\title{
Velocity Fields in Spiral Galaxies ${ }^{1}$
}

\author{
A.M. Fridman ${ }^{2}$, O.V. Khoruzhii ${ }^{3}$ \\ Institute of Astronomy, Russian Academy of Sciences, 48 Pyatnitskaya \\ St., Moscow, 109017, Russia
}

Abstract. Discussed are various observable features of velocity fields in spiral galaxies. Main attention is paid to giant anticyclones and motions perpendicular to the disk plane.

\section{Introduction}

There are two different observable manifestations of the density waves in gaseous disks of spiral galaxies. The first is well-known spiral arms - a periodical structure in the surface density. The second is a specific velocity field of a gas.

In this work we consider two features of the velocity field of a density wave. First, giant anticyclones in the disk plane located near the corotation have been discussed more then ten years beginning from the paper by Nezlin et al. (1986). Second, the motions along the rotation axis are only beginning to be discussed (Fridman et al.1998).

One can show that the anticyclones jointly with spiral arms compose a unique spiral-vortex structure. It means, in particular, that using the theory of the spiral density waves one may restore a full three-component velocity field of the gas from observed one-component line-of-sight velocity. Indeed, a method was worked out, which gave the possibility to find the velocity field in the disk plane and independently by several ways to determine the corotation radius. It offered to discover the giant anticyclones near the corotation circle in full accordance with the theory (Lyakhovich et al. 1997, Fridman et al 1997). The method include also the observational check of its basic assumption, that the spiral arms observed represent the density wave in the galaxy.

The same method was applied to investigate vertical motions of a gaseous galactic disk.

Two types of vertical motions in galactic disks are known (Fridman and Polyachenko 1984): "membrane" motions ("bending oscillations") and vertical motions in the density wave, which we shall call "vertical spiral-wave" motions.

\footnotetext{
${ }^{1}$ This work was performed under the partial support of RFBR grants N 96-02-17792 and N 96-02-19636, grant "Leading Scientific Schools" N 96-15-96648, and the grant "Fundamental Space Researches. Astronomy" for the 1998 year N 1.2.3.1, N 1.7.4.3.

${ }^{2}$ also Stemberg Astronomical Institute, Moscow State University, Moscow 119899, Russia

${ }^{3}$ also National Research Center of Russian Federation "Troitsk Institute for Innovation and Fusion Researches", Troitsk, Moscow Reg. 142092, Russia
} 
These two types of motions have different dispersion properties (Fridman and Polyachenko 1984) and symmetries about the $z=0$ plane. The velocities of membrane oscillations are an even function of $z(v(z)=v(-z)$ ), while the velocities of vertical spiral-wave motions are an odd function of $z(v(z)=-v(-z))$. Up to the current time, the main method for studying membrane oscillations has been obtaining observations of warps in galactic disks. The most convenient galaxies for such observations are those viewed nearly edge-on. However, in this case, it is not possible to say anything about the velocities in these perturbations, i.e., about their dynamical properties.

In the case of density waves, studies of vertical motions are impeded by their relative smallness (as a consequence of the smallness of the parameter $k h$, where $k$ is the absolute value of the wave vector and $h$ is the disk halfthickness). Therefore, their contribution to the observed radial velocities in galaxies is relatively important only for galaxies that are viewed nearly faceon, when the contribution of motions in the plane of the disk is suppressed (proportional to $\sin i$, where $i$ is an inclination angle of the galaxy). Thus, studies of both types of vertical motions require analyses of the velocity fields in galaxies that are viewed face-on.

Another important circumstance is associated with the fact that, determining the vertical $(z)$ component of the velocity of some part of a disk viewed face-on, we determine the "mean" velocity of this section over the entire "visible" thickness of the disk. If the optical depth of the disk at the wavelength considered is modest (as is true for the 21-cm line), the contributions of the near and far halves of the disk to the observed line-of-sight velocity are comparable. In this case, the observed amplitude of spiral-wave motions should be small, since the motion on different sides of the $z=0$ plane displays mirror symmetry (if the optical depth is zero, the mean velocity is zero - the contributions of the two halves precisely compensate each other). In the case of a large disk opacity, as in the $\mathrm{H}_{\alpha}$ line, the amplitude of spiral-wave motions will be substantially greater, since we effectively measure the velocity of the nearby outer boundary of the disk, where this velocity is maximum (the vertical velocity of the gas in the density wave grows with $z$ ). The velocities of vertical motions in membrane oscillations are virtually constant throughout the disk thickness (due to the smallness of the parameter $k h$ ); therefore, the ratio of the velocity amplitudes measured at optical and radio wavelengths should not depend significantly on the difference in the optical depths in these two observing bands. In this way, comparing the velocity fields for a galaxy viewed face-on derived using optical and radio data makes it possible to draw conclusions, not only about the velocity distribution for vertical motions, but also about the origin of these motions.

\section{Giant anticyclones in spiral galaxies}

To discover predicted anticyclones in spiral galaxies we worked out a method to restore the total three-component vector velocity field of the gas in a spiral galaxy from the observed (one-component) field of the line-of-sight velocities (Lyakhovich et al.1997, Fridman et al.1997 a, b). Evidently, it would be impossible without making some assumptions. The method uses two assumptions. 

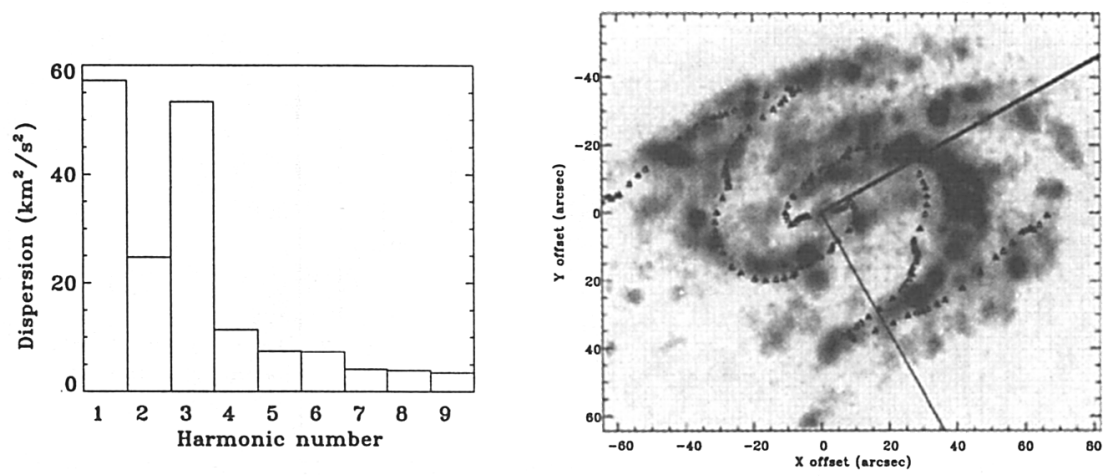

Figure 1. Left. Contribution of different Fourier harmonics of lineof-sight velocity field of NGC 157 in dispersion in the model of pure circular motion. The prevalence of first sine, second and third harmonics is clearly seen and demonstrates the domination of the mode with $m=2$ in velocity perturbations in the disk. Right. Superposition of the modified third harmonic of the line-of-sight velocity field on the $H_{\alpha}$ image of NGC 157 (grey scale). The modified third harmonic has a form $\cos \left(2 \varphi+\pi / 2-F_{3}\right)$, where $F_{3}$ is the phase of the original third harmonic. Triangles show the azimuth positions of the maxima of the harmonic at each radius. A good correspondence of the phase curve to the observed position of the spiral arms gives strong evidence for the non-circular velocities to be associated with the spiral structure and proves a wave nature of the spiral structure in this galaxy.
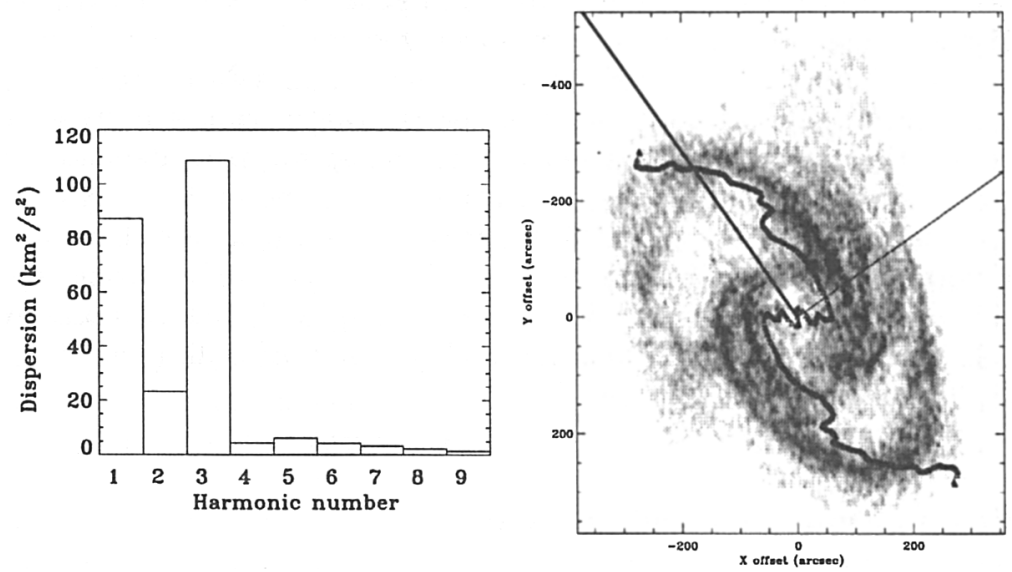

Figure 2. The same as in Fig. 2 but for the galaxy NGC 1365. The modified third harmonic is overlaid on HI image of the galaxy. The velocity field and $\mathrm{HI}$ image were kindly provided to us by P.O. Lindblad, description of the data see Lindblad, Lindblad, \& Athanassoula 1996. 

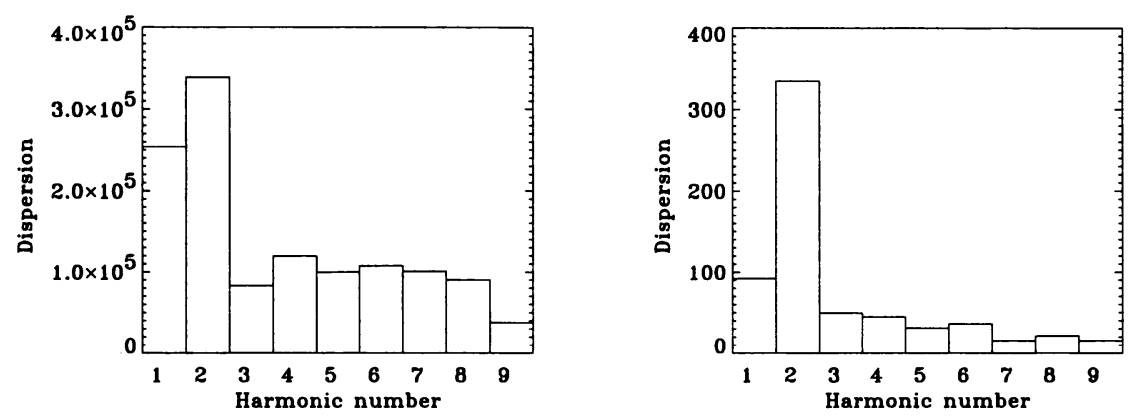

Figure 3. Contribution of different Fourier harmonics of the brightness into deviations from axisymmetry in the disks of NGC 157 (left) and NGC 1365 (right). $\mathrm{H}_{\alpha}$ line image is used for NGC 157, and 21-cm line image is used for NGC 1365. The prevalence of second harmonic is clearly seen.

First, the observed perturbations of different disk parameters (density, velocity etc.) are not independent from each other, but are different manifestations of the unique self-consistent density wave. Second, in the density wave a mode with particular azimuthal number $m$ corresponding to observed number of spiral arms dominates. The latter allows to approximate components of velocity perturbations in a simple form $V_{i}=C_{i} \cos \left(m \varphi-F_{i}\right)$, where $C_{i}$ is an amplitude, and $F_{i}$ is a phase. Remarkably, both assumptions can be checked directly from the observations (Figs. 1, 2 and 3, for details see Lyakhovich et al.1997, Fridman et al.1997 a, b). The restoration of the velocity field automatically allows determination of the corotation radius of the density wave. The method was successfully applied to several grand design galaxies: NGC 157, NGC 3893, NGC 1365 and some others. Fig. 4 shows the velocity field in the plane of the disk in the corotating frame of reference for the galaxies NGC 157 (left) and NGC 1365 (right). In both cases we can see two anticyclones between spiral arms. The location of anticyclones relative to the spiral arms depends on the behaviour of basic parameters in the corotation region and on the mechanism of the wave generation (Nezlin et al.1986, Baev et al.1987, Lyakhovich et al.1996). Centres of vortices lie between spiral arms in the case both of a strong centrifugal instability, when $\kappa^{2} \equiv 4 \Omega_{0}^{2}\left(1+r \Omega^{\prime} / 2 \Omega\right)<0,(e . g$. Mrk 1040, Afanasiev and Fridman 1993), and of a gravitational instability, when $\kappa^{2}>0$, (e.g. NGC 157, NGC 3893, Fridman et al.1997b, 1999).

\section{Vertical motions in spiral galaxies}

To analyse vertical motions associated with density waves we chose the galaxy NGC 3631 (type SA(s)c), which has two main, rather long, spiral arms and is oriented nearly face-on $\left(i=17^{\circ}\right)$. The domination of the two-armed mode is clearly seen in Fourier decomposition of the brightness of the disk both in $\mathrm{H}_{\alpha}$ and 21-cm lines (Fig. 5). There are no objects of comparable brightness near this 

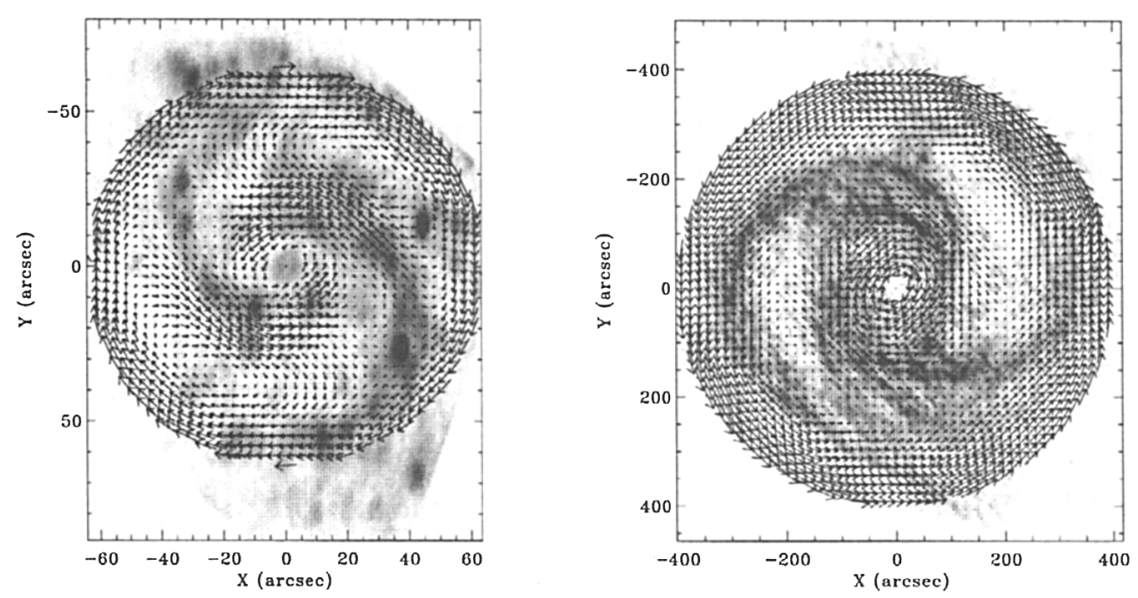

Figure 4. Restored velocity fields of NGC 157 (left) and NGC 1365 (right) in the reference frame rotating with the pattern speed overlaid on the deprojected $H_{\alpha}$ and $\mathrm{HI}$ images of the galaxies respectively.

galaxy, which makes it improbable that the galactic velocity field is distorted by tidal forces.

The HI observations of NGC 3631 used for the study were obtained by Knapen (1997) using the Westerbork Synthesis Radio Telescope, and have angular resolution (FWHM of the synethesized beam) 15." $2 \times 11^{\prime \prime}$.

Optical observations were obtained on the 6-m telescope of the Special Astrophysical Observatory (Fridman et al.1998). The initial velocity field derived from $\mathrm{H}_{\alpha}$ observations has angular resolution $2^{\prime \prime}$. In order to facilitate the comparison with the radio observations, we smoothed the optical observations with a Gaussian with half-width (HWHM) of $6 .{ }^{\prime \prime} 5$, which is close to the resolution of the radio observations.

In order to conduct a quantitative comparison of the velocity fields using a single method, we performed a Fourier analysis of the distribution of the lineof-sight velocities in azimuth angle in the plane of the galactic disk (measured from the line of nodes), using the "Vortex" program developed at the Institute of Astronomy (Lyakhovich et al. 1997). The size of the disk region studied ( $R$ $<89^{\prime \prime}$ ) was restricted to the size of the velocity field determined by the optical data. The inclination angle of the galactic disk and the position angle of the line of nodes were taken to be the same for the optical and radio data, and to be constant over the entire region studied: $i=17^{\circ}, \mathrm{PA}=150^{\circ}$ (see Knapen 1997, Table 3).

In both the optical (Fig. 6a) and radio (Fig. 6c) velocity fields, the spectra are dominated by the first three Fourier harmonics, as is natural, given the twoarmed spiral structure of the galaxy (Smirnov and Sakhibov 1987, 1989, 1990; Canzian 1993; Lyakhovich et al. 1997; Fridman et al. 1997b). Motions in the galactic disk called forth by the two-armed density wave lead to the appearance of the first and third harmonics in the spectrum. The nature of the second Fourier harmonic is not entirely clear. It could be associated with asymmetry in 

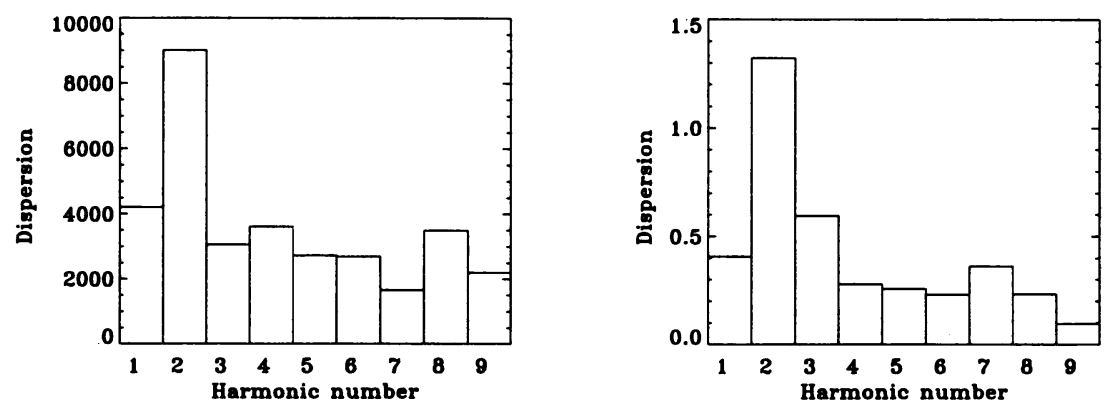

Figure 5. Contribution of different Fourier harmonics of the brightness in $\mathrm{H}_{\alpha}$ line (left), and 21-cm line (right) into deviations from axisymmetry in the disk of NGC 3631 . The prevalence of second harmonic is clearly seen.

the two-armed structure (i.e., an appreciable contribution of an $m=1$ mode) or due to vertical motions in the two-armed density wave (Lyakhovich et al. 1997, Fridman et al. 1997b). The good correlation between the lines of the maxima of the second harmonic and the shape of the spiral patterns observed for a number of galaxies (Fridman et al. 1999) argues in favor of this latter interpretation.

The results presented in Fig. 6 are consistent with the hypothesis that the second Fourier harmonic in the line-of-sight velocity field spectrum of NGC 3631 is associated with "vertical spiral-wave" gas motions (see Introduction). Indeed, in the case of the optical data, only the second harmonic has a significant amplitude, which can easily be understood if precisely this harmonic is associated with vertical motions, while the effect of projection decreases the contributions of the radial and azimuthal velocities, which are proportional to $\sin i$ (in the case of NGC 3631, by more than a factor of three: $\sin 17^{\circ}=0.3$ ).

At the same time, the amplitude of the second harmonic for the radio data is substantially lower than that for the optical data, which is natural according to Introduction, taking into account the lower HI optical depth. Note that the galactic disk is not completely transparent in the HI line. The inhomogeneity of the atomic hydrogen in temperature and density and the different coverages in and between the spiral arms (Brown 1997) hinders quantitative estimation of the effects of absorption.

The radial behavior of the amplitudes of the third harmonic (recalculated for the plane of the galaxy) is presented in Fig. 7, which also shows lines of constant phase corresponding to the maximum deviations. It is noteworthy that the phase behavior of the optical and radio harmonics is similar, despite the difference in their amplitudes. This suggests that the radio and optical harmonics result from a single mechanism, evidently associated with the spiral density wave.

As shown by Fridman et al. (1997), the relation between the observed third harmonic and the spiral pattern can be verified using so-called "modified" third harmonic. The latter is a two-armed spiral with phase $F_{3}-\pi / 2$, i.e., $\propto \cos \left(2 \varphi-F_{3}+\pi / 2\right)$, where $F_{3}$ is the phase of the original third harmonic of 


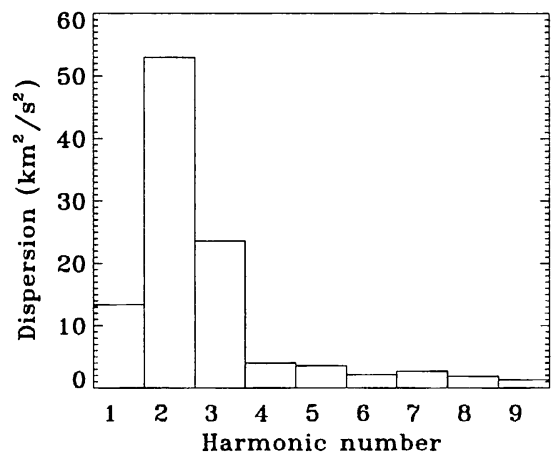

a)

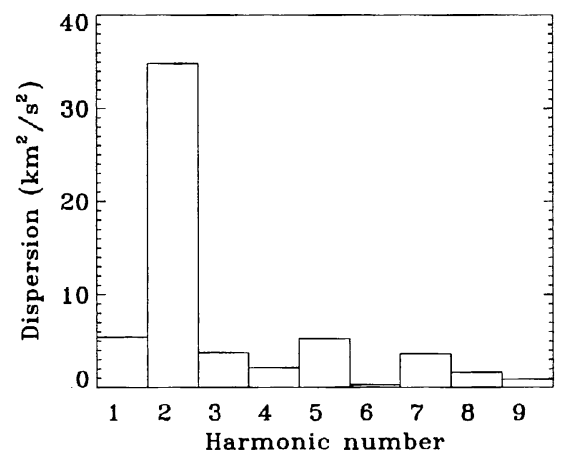

b)

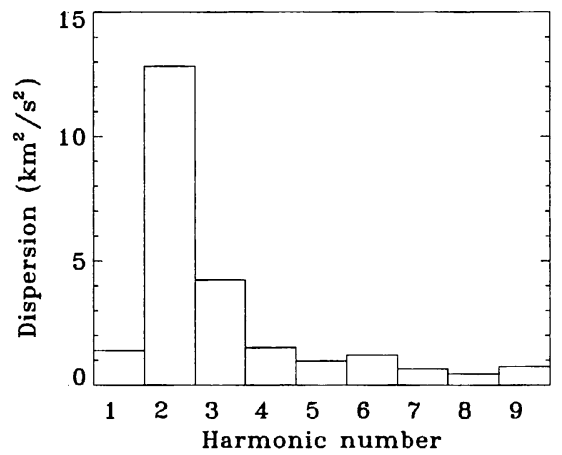

c)

Figure 6. Contribution of different Fourier harmonics of line-of-sight velocity field of NGC 3631 in dispersion in the model of pure circular motion: (a) calculated from initial $\mathrm{H}_{\alpha}$ velocity field; (b) calculated from smoothed $\mathrm{H}_{\alpha}$ velocity field; (c) calculated from $\mathrm{HI}$ velocity field. The prevalence of first sine, second and third harmonics is clearly seen and demonstrates the domination of the mode with $m=2$ in velocity perturbations in the disk. 

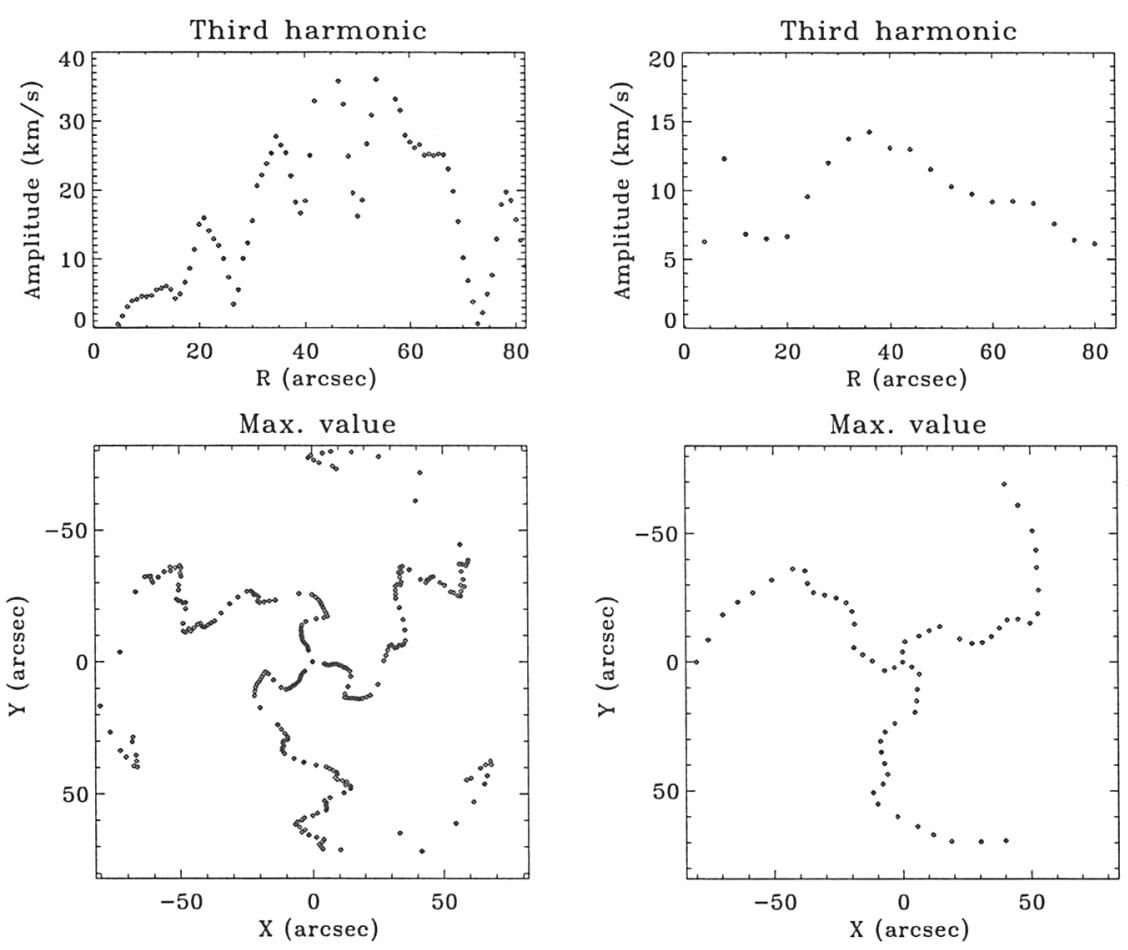

Figure 7. Radial behaviour of the amplitude (top) and the phase (bottom) of third Fourier harmonics of line-of-sight velocity. (left) $\mathrm{H}_{\alpha}$ velocity field. (right) HI velocity field. 

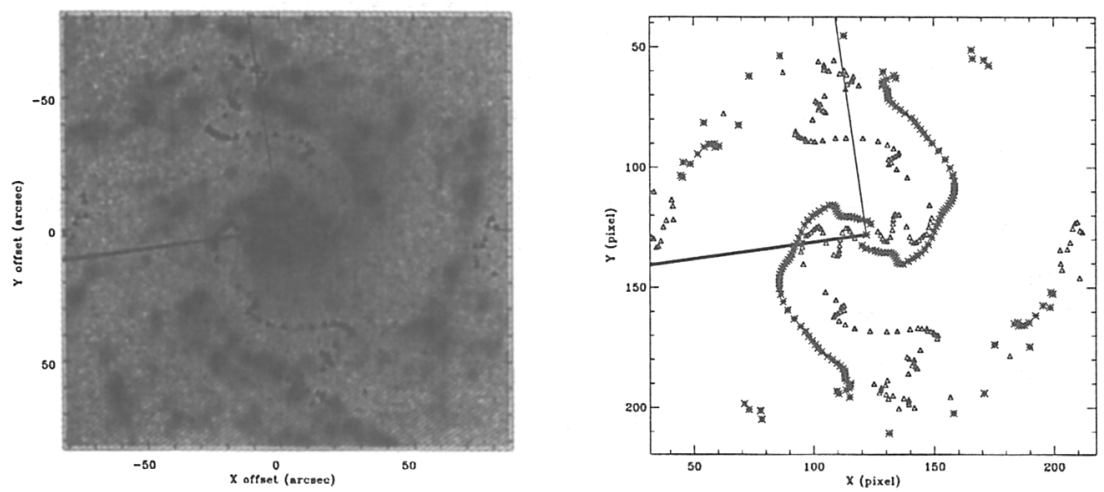

Figure 8. Superposition of the modified third harmonic of the lineof-sight velocity field of NGC 3631 on the galaxy $H_{\alpha}$ image (left) and on the lines of constant phase of the second harmonics of brightness (right). Triangles and asterisks respectively show the azimuth positions of the maxima of the harmonics at each radius.

the line-of-sight velocity field. Fig. 8 shows lines of constant phase for the modified third harmonic, corresponding to the maximum deviations, superposed on a monochromatic image of NGC 3631 in the $\mathrm{H}_{\alpha}$ line. We can clearly see a correlation between the phase of the spiral pattern and the modified third harmonic. As shown by Fridman et al. (1997) this correlation proves the wave nature of the spiral structure of the galaxy. It is noteworthy that, out to distances from the center of $R \approx 40 \div 50^{\prime \prime}$, the modified harmonic is shifted from the spiral pattern by $\pi / 2$, while at large distances from the center, it lies on the regions of maximum brightness. This may be due to a change in the relation between the phases of the spiral pattern and modified third harmonic near the corotation radius of the spiral structure (Fridman et al. 1997). Consequently, these data suggest that the corotation radius of NGC 3631 is located near $40 \div 50^{\prime \prime}$.

Acknowledgments. We are grateful to Per Olaf Lindblad who kindly provided to us the velocity field of NGC 1365 .

\section{References}

Afanasiev, V.L., \& Fridman, A.M. 1993, Astron. Lett., 195, 319

Baev, P.V., Makov, Yu.N., \& Fridman, A.M. 1987, Sov. Astron. Lett., 13, 406

Brown, R. 1997, ApJ, 484, 637

Canzian, B. 1993, Astron. Soc. Pacif. Conf. Series, 105, 661

Fridman, A.M., Khoruzhii, O.V., Lyakhovich, V.V., Avedisova, V.S., Sil'chenko, O.K., Zasov, A.V., Rastorguev, A.S., Afanasiev, V.L., Dodonov, S.N., \& Boulesteix, J. 1997a, The Twenty-first International Conference on the Unity of the Sciences, Washington, November 24-30, 1997 
Fridman, A.M., Khoruzhii, O.V., Lyakhovich, V.V., Avedisova, V.S., Sil'chenko, O.K., Zasov, A.V., Rastorguev, A.S., Afanasiev, V.L., Dodonov, S.N., \& Boulesteix, J. 1997b, Astroph. and Space Sci., 252, 115

Fridman, A.M., Khoruzhii, O.V., Zasov, A.V., Sil'chenko, O.K., Burlak, A.N., Moiseev, A.V., Afanasiev, V.L., Dodonov, S.N., \& Knapen, J. 1998, Astron. Lett., 24, 764

Fridman, A.M., Khoruzhii, O.V., Lyakhovich, V.V., Sil'chenko, O.K., Zasov, A.V., Afanasiev, V.L., Dodonov, S.N., \& Boulesteix, J. 1999, MNRAS, in press

Knapen, J.H. 1997, MNRAS, 286, 403

Lindblad, P.A.B., Lindblad, P.O., \& Athanassoula E. 1996, A\&A, 313, 65

Lyakhovich, V.V., Fridman, A.M., Khoruzhii, O.V., \& Pavlov, A.I. 1997, Astronomy Report, 41, 447

Nezlin, M.V., Polyachenko, V.L., Snezhkin, E.N., Trubnikov, A.S., \& Fridman, A.M. 1986, Sov. Astron. Lett., 12, 213

Sakhibov, F.Kh., \& Smirnov, M.A. 1987, Astron. Zh., 64, 255

Sakhibov, F.Kh., \& Smirnov, M.A. 1989, Astron. Zh., 66, 921

Sakhibov, F.Kh., \& Smirnov, M.A. 1990, Astron. Zh., 67, 690 